\title{
ENDOSCOPY
}

\section{You get what you expect? A critical appraisal of imaging methodology in endosonographic cancer staging}

\author{
A Meining, H J Dittler, A Wolf, R Lorenz, V Schusdziarra, J-R Siewert, M Classen, \\ H Höfler, T Rösch
}

See end of article for authors' affiliations ......................

Correspondence to: Dr T Rösch, Department of Internal Medicine II, Technical University of Munich, Klinikum rechts der Isar, Ismaningerstrasse 22, D-81675 München, Germany;

Thomas.Roesch@

Irz.tu-muenchen.de

Accepted for publication 21 August 2001

\begin{abstract}
Background and aims: After an initial period of excellent results with newly introduced imaging procedures, the accuracy of most imaging methods declines in later publications. This effect may be due to various methodological factors involved in the research. Using the example of endoscopic ultrasound (EUS), this study aimed to elucidate one of the factors possibly concerned-namely, the extent to which the examiners are adequately blinded.

Methods: Well documented videotapes of EUS examinations of 101 patients with resected tumours of the oesophagus $(n=32)$, stomach $(n=33)$, or pancreas $(n=36)$ were evaluated in three different ways: firstly, retrospective analysis under routine clinical conditions; secondly, evaluation of EUS videotapes in a strictly blinded fashion; and thirdly, evaluation of the same videotapes but with additional information from the video endoscopic appearance (oesophageal/gastric cancer) or from computed tomography results (pancreatic cancer). Histopathological T staging was used as the reference method.

Results: The accuracy of EUS in T staging was $73 \%$ under routine conditions. This value fell significantly to $53 \%$ for the blinded evaluation but increased again to $62 \%$ for the unblinded evaluation. The sensitivity of staging T1/T2 tumours was 72\% (routine EUS), $59 \%$ (blinded EUS), and $70 \%$ (unblinded EUS). The respective values for advanced tumours were $85 \%, 74 \%$, and $72 \%$.

Conclusions: The accuracy of EUS for T staging in clinical practice appears to be lower than has previously been reported. In addition, blinded analysis produced significantly poorer results, which improved when another test was added. It may be speculated that better results with routine EUS obtained in a clinical setting are due to additional sources of information.
\end{abstract}

A ter each new imaging procedure is introduced, scientific evaluations of it yield excellent results; afterwards, however, wider application of the method-and moderation of the initial enthusiasm for it-tend to lead to more variable, and often considerably less impressive, accuracy rates. One recently introduced imaging procedure, namely endoscopic ultrasound (EUS), has been widely evaluated for use in the locoregional staging of gastrointestinal tumours. Particularly during the initial phase of assessment, many studies reported a clear advantage of EUS over other imaging methods such as computed tomography (CT) scanning, magnetic resonance imaging (MRI), and conventional ultrasonography. ${ }^{12}$ EUS has been described as highly accurate, and as less invasive and more cost effective than other imaging methods for local staging of oesophagogastric and pancreatic cancers. ${ }^{134}$

However, in more recent reports, the accuracy of EUS has

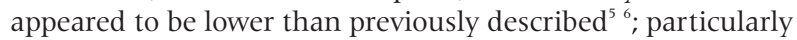
in the locoregional staging of pancreatic cancer, the results of EUS alone and of EUS in comparison with helical CT have been variable. ${ }^{48}$ The explanations for these discrepancies may lie to some extent in differences in the methodologies of the studies concerned. The aim in the present study was to better characterise these methodological factors by evaluating the tumour staging accuracy of EUS in a single group of patients under different assessment conditions. Routine results obtained in the clinical setting were compared with a strictly blinded "scientific" approach, with and without additional imaging information.

\section{PATIENTS AND METHODS}

A total of 101 patients with tumours of the oesophagus $(\mathrm{n}=32)$, stomach $(\mathrm{n}=33)$, and pancreas $(\mathrm{n}=37)$ over a five year period (1992-1996) were analysed retrospectively.
Inclusion criteria were:

(1) Availability of well documented videotapes of EUS examinations for re-evaluation, with visualisation of the entire tumour on instrument withdrawal-for example, with normal wall being visible distal (if the tumour was traversable) and proximal to the tumour mass in cases of gastrointestinal cancer and mass lesion, and the portal system in cases of pancreatic cancer. This selection was made by an independent experienced EUS examiner (RL) who was not involved in the tape evaluation for this study.

(2) Availability of endoscopic videotape sequences of oesophageal and gastric cancers, also showing complete tumour visualisation.

(3) Availability of CT results in patients with pancreatic cancer.

(4) Availability of initial clinical EUS reports (with definition of the T category).

(5) Patients had to have undergone complete tumour resection, with data on the histopathological $\mathrm{T}$ staging available to serve as the reference method (with one exception in a case of gastric cancer; only stages Tl-T3 were therefore ultimately included).

The data were analysed in three different ways:

(1) Routine analysis. A retrospective analysis of the T staging results from the EUS reports produced at the initial EUS examinations in routine clinical conditions.

Abbreviations: $\mathrm{CT}$, computed tomography; EUS, endoscopic ultrasonography; MRI, magnetic resonance imaging. 


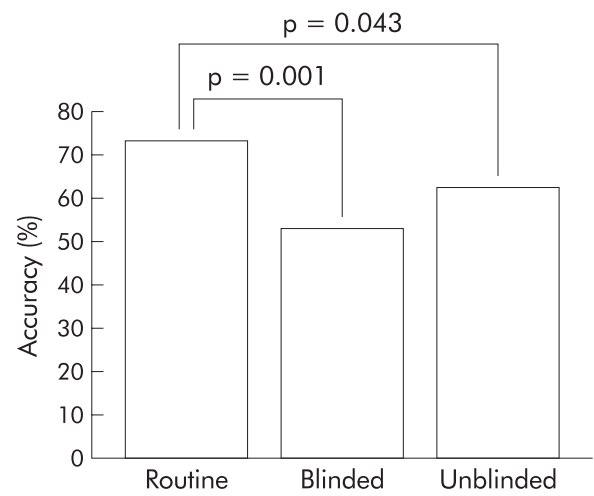

Figure 1 Overall accuracy of T staging in routine examinations compared with blinded and unblinded re-evaluation ( $\mathrm{McNemar}$ test). No statistically significant difference between the blinded and unblinded analysis was found.

(2) Blinded analysis. The videotapes recorded at the initial examination were re-evaluated by one of the main investigators (TR). Patients were mixed, and their names were concealed. This analysis was performed a minimum of 18 months (range 18-72 months) after the initial routine examination. At this time, the only information available to the examiner was the anatomical site of the tumour. Other data, such as the patient's name, age, sex, and clinical findings, were not revealed.

(3) Unblinded analysis. A minimum of a further 18 months (range 18-24 months) after the blinded re-evaluation, the videotapes recorded at the initial examination were evaluated once again. However, this time the investigator was not blinded, and was allowed to review the corresponding endoscopy tapes before the EUS assessment (for oesophagogastric cancers) or to read the CT reports (for pancreatic cancers). Other clinical data (including patient names), and any imaging information other than the endoscopy or CT data, were still not made available.

EUS examinations were done in a standardised way by all examiners by scanning the tumour extent after passage to the distal tumour free part of the organ and then slowly withdrawing the instrument along the tumour axis; similarly, established and previously published criteria for EUS staging were used. ${ }^{9}$ Briefly, oesophagogastric tumours mostly appeared as echo poor localised or circumferential wall thickening, with progressive loss of the underlying wall layer structure corresponding to the tumour stage. Stage Tl was thus assumed on EUS if the lesion was limited to the mucosa and submucosa (first three layers). Stage T2 was assumed if the tumour infiltrated the entire wall, leading to complete or nearly complete loss of the layer structure, but with a smooth outer margin and/or small parts of the muscularis layer (fourth layer) being recognisable. In contrast, the echo poor wall thickening leading to an endosonographic diagnosis of stage T3 showed irregular outer margins, with clear infiltration of the adventitia and surrounding fat tissue. In stage T4, infiltration of other organs had to be visualised. For pancreatic cancer, the old TNM system (1987) was used: stage T1 was assumed on EUS if the tumour was limited to the pancreas, and stage $\mathrm{T} 2$ if it had spread beyond the pancreas but was not infiltrating major parapancreatic vessels, as was the case when stage T3 was diagnosed. Criteria for vascular infiltration were total or partial vascular obstruction, with or without collateral vessels, visualisation of tumour in the vessel, or a grossly irregular tumour-vessel interrelationship.

The results obtained using all three assessment methods were compared with the histopathological findings for the resected tumours, which served as the gold standard. Only resected cases and those pancreatic cancers with thorough surgical exploration were included in order to have a reliable and uniform gold standard.

To assess interobserver variability as a potentially confounding factor, oesophagogastric tumour assessments for the blinded and unblinded analyses were repeated by a second independent examiner (HJD) under the same conditions. Both evaluators (TR and HJD) had experience of more than 500 oesophagogastric and more than 250 pancreatic EUS examinations at the time of the study.

Accuracy rates were calculated for all three methods. In addition, the sensitivity of EUS for detecting less invasive (Tl/2) or advanced tumours (T3/T4) was determined. Differences between the respective groups were tested for statistical significance using the McNemar test for paired samples. A p value $<0.05$ was considered significant.

\section{RESULTS}

After screening of the relevant videotape sequences from the study period, a total of 101 patients were identified who met the entry criteria. The results for the three assessment methods showed an overall T staging accuracy of $73.3 \%$ (74 of 101) for routine EUS examinations which was significantly better than the rate of $52.5 \%$ ( 53 of 101 ) for the blinded evaluation of the videotapes and $62.4 \%$ (63 of 101) for the unblinded re-evaluation of the videotapes (McNemar test: $p=0.001$ and $\mathrm{p}=0.043$, respectively) (fig 1). However, the "unblinded" evaluation involved adding information from only one other imaging test (endoscopy or CT) but no other clinical or imaging data. The information available in clinical routine practice may therefore still contain substantially more data than provided in this "unblinded" analysis.

When the data for localisation of tumours were stratified, it was found that blinded evaluation of the EUS videotapes was associated with a reduced accuracy for all three tumour sites, reaching statistical power for the oesophagus and stomach. Although the accuracy rates for pancreatic and oesophageal cancers improved significantly after the investigator was "unblinded" before re-evaluating the videotapes, no such effect was seen for gastric tumours (table 1). This low level of accuracy in gastric cancer even when the examiner was not blinded involved overstaging of the tumours in most cases ( 14 of 20). Overstaging was particularly frequent with pT2 tumours (nine of 12) and those located in the proximal stomach (six of eight).

The accuracy rates for the various $\mathrm{T}$ stages ( $\mathrm{Tl}-\mathrm{T} 3$ ), as evaluated by the three methods, are shown in table 2 . Only one

\begin{tabular}{|c|c|c|c|c|}
\hline & $\begin{array}{l}\text { All patients } \\
(\mathrm{n}=101)\end{array}$ & $\begin{array}{l}\text { Oesophageal } \\
\text { cancer }(n=32)\end{array}$ & $\begin{array}{l}\text { Gastric cancer } \\
(\mathrm{n}=33)\end{array}$ & $\begin{array}{l}\text { Pancreatic cancer } \\
(\mathrm{n}=36)\end{array}$ \\
\hline Routine analysis & $73.3 \%$ & $81.3 \%$ & $66.7 \%$ & $72.2 \%$ \\
\hline Blinded & $52.5 \%$ ** & $50.0 \%$ ** & $45.5 \%$ * & $61.1 \%$ \\
\hline Unblinded & $62.4 \% *$ & $71.9 \%$ & $39.4 \%$ ** & $75.0 \%$ \\
\hline
\end{tabular}


Table 2 Accuracy of T staging, stratified according to $\mathrm{pT}$ stages $\mathrm{Tl}-\mathrm{T3}$

\begin{tabular}{lllll}
\hline & $\begin{array}{l}\text { All patients } \\
(\mathrm{n}=100)\end{array}$ & $\begin{array}{l}\mathrm{pT1} \\
(\mathrm{n}=10)\end{array}$ & $\begin{array}{l}\mathrm{pT2} \\
(\mathrm{n}=44)\end{array}$ & $\begin{array}{l}\mathrm{pT} 3 \\
(\mathrm{n}=46)\end{array}$ \\
\hline Routine analysis & $73.0 \%$ & $60.0 \%$ & $63.6 \%$ & $84.8 \%$ \\
Blinded & $52.0 \% * *$ & $60.0 \%$ & $43.2 \% *$ & $58.7 \% *$ \\
Unblinded & $63.0 \% *$ & $40.0 \%$ & $61.4 \%$ & $69.6 \% *$ \\
\hline
\end{tabular}

* $p<0.05,{ }^{* *} p<0.01$ compared with "routine analysis" (McNemar test).

Table 3 Sensitivity of endoscopic ultrasonography for detecting $\mathrm{pT} 1 / \mathrm{T} 2$ cancers versus $\mathrm{pT} 3 / \mathrm{T} 4$ cancers

\begin{tabular}{lll}
\hline & pT1/T2 (n=54) & pT3/T4 (n=47) \\
\hline Routine analysis & $72.2 \%$ & $85.1 \%$ \\
Blinded & $59.3 \% *$ & $74.5 \% \dagger \dagger \dagger$ \\
Unblinded & $70.4 \%$ & $72.3 \% \dagger \dagger \dagger$ \\
\hline
\end{tabular}

" $p<0.05$ compared with "routine analysis" and "unblinded" (McNemar test); $† \dagger \dagger p<0.001$ compared with "routine analysis" (McNemar test).

patient with a gastric cancer had a tumour in stage pT4 (he underwent palliative resection) which was correctly staged in the routine EUS examination and in the blinded evaluation of the tapes, and was understaged as a T3 tumour after unblinded re-evaluation.

When the sensitivity of EUS for detecting tumours limited to the wall (T1/T2) was compared with its sensitivity for detecting advanced tumours (T3/T4), it was found that all three methods had better sensitivity rates in staging advanced lesions. However, both blinded and unblinded re-evaluation of the videotapes led to lower accuracy rates compared with those obtained in the routine analysis. This difference proved to be significant for both groups in relation to stage T3/T4 tumours. For non-invasive stage $\mathrm{T} 1 / \mathrm{T} 2$ tumours, only the blinded analysis resulted in a significantly lower level of accuracy (table 3 ).

In patients with oesophageal tumours, excluding those who had a non-traversable tumour stenosis $(n=13)$, did not lead to improved accuracy with EUS (routine analysis 78.9\% v 81.3\%; blinded analysis $42.1 \% \vee 50.0 \%$; unblinded analysis $68.4 \% v$ $71.9 \%)$.

A second examiner (HJD) was involved in reviewing the videotapes for the staging of oesophagogastric tumours, and his findings were compared with those of the main investigator (TR); the same effects were observed. The respective kappa values for testing interobserver variability between the two examiners were 0.264 (blinded) and 0.528 (unblinded).

Examples of oesophageal cancer staging are shown in figs 2 and 3

\section{DISCUSSION}

The main aim of the present paper was not to present accuracy or outcome data on endosonographic cancer staging but to further analyse some of the methodological factors involved in scientific publishing concerning the accuracy of imaging tests. We speculated that one of those factors may be additional information being fed into the seemingly "pure" interpretation of the test results. This could be due to the simple fact that the investigators are familiar with the patients concerned and their clinical features, indicating advanced disease; the results of blood tests, previous endoscopic findings, or results previously obtained from other tests are usually known before an examination is begun. While this total view of the patient and his or her findings, taking into account every aspect of the

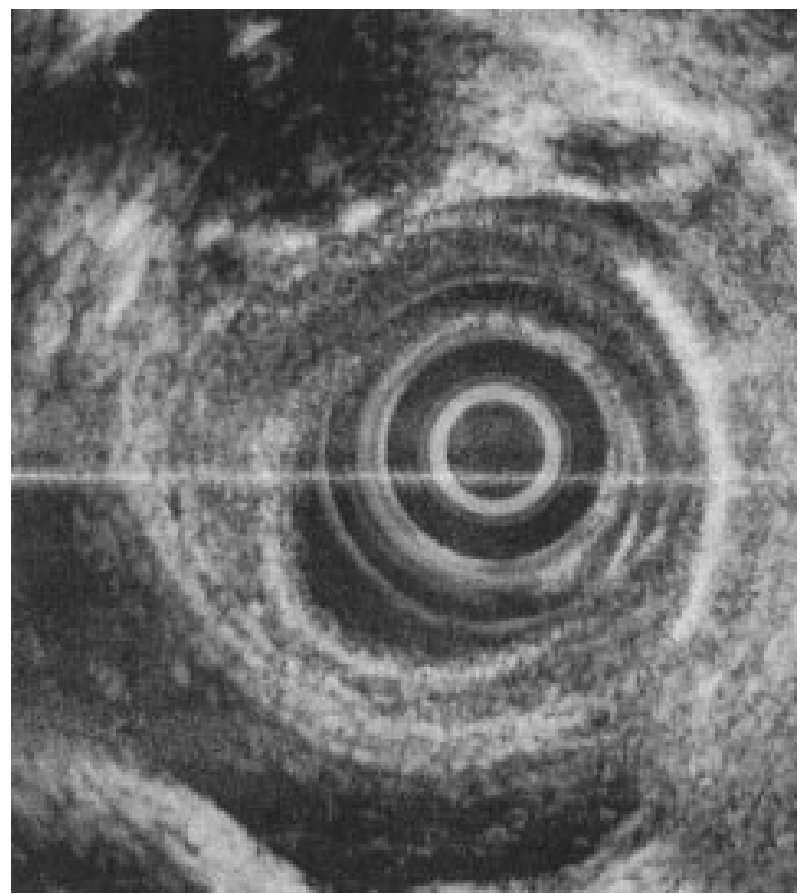

Figure 2 An oesophageal carcinoma in histopathological stage T2 but with endosonographic visualisation of transmural tumour growth, falsely suggesting stage T3 on endoscopic ultrasonography.

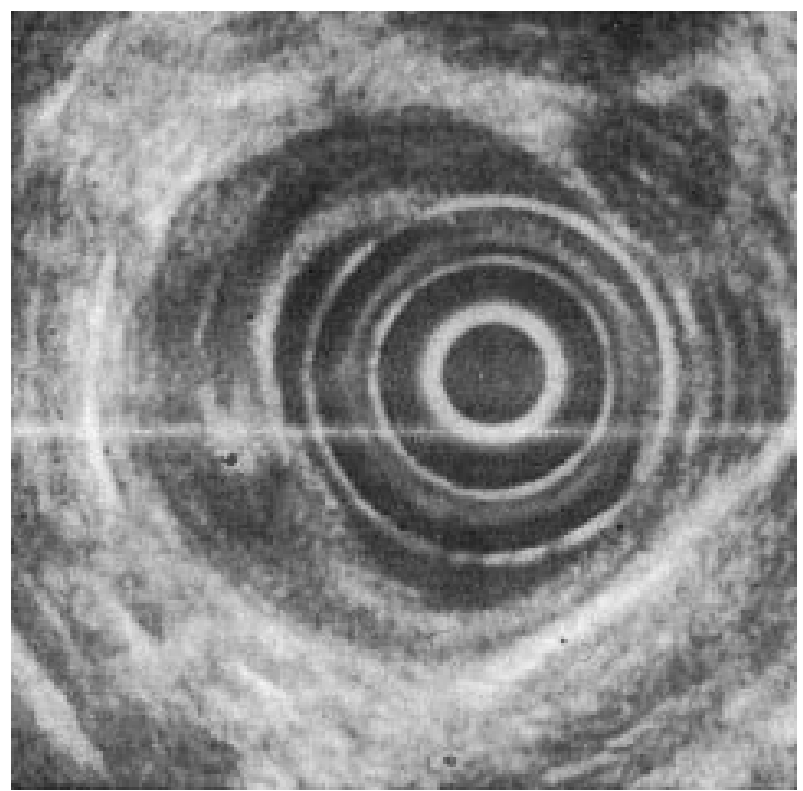

Figure 3 An oesophageal carcinoma in histopathological stage T3, similarly demonstrating transmural tumour growth, this time correctly suggesting stage T3.

disease, is an excellent approach for clinical case management, it is problematic during scientific evaluation of a specific procedure or subset of procedures when the aim should be to have a minimum of confounding factors. Particularly when the results of imaging tests are contradictory in clinical practice, the "real" accuracy of each procedure needs to be known independently of other procedures.

There is ample evidence of the phenomenon of initial good results which are not maintained in later publications, both in the area of EUS - the topic of the present study-and also with other imaging procedures. This was recently shown for EUS in pancreatic cancer staging. ${ }^{5-7}$ In addition, comparisons 
between EUS and helical CT in the clinical staging of pancreatic cancer have yielded conflicting results. ${ }^{4}{ }^{10-12}$ Of the potentially responsible methodological factors, blinding towards other imaging tests is a criterion rarely applied. ${ }^{5}{ }^{13}$ In fact, it is quite difficult in a clinical setting to keep an examiner strictly blinded to the results of other tests. However, the same is also true of other imaging procedures-and therefore this also applies to published studies of CT and MRI in the locoregional staging of pancreatic cancer, for example. In the late 1980s and early 1990s, conventional (that is, non-helical) CT obtained accuracy rates of approximately $90 \%$ in a fair number of studies $^{14}{ }^{15}$; similarly, high accuracy rates were again reached with helical $\mathrm{CT}^{81116}$ which was then treated as the gold standard. However, when abdominal MRI became more firmly established at the end of the 1990s, MRI was found to be approximately $90 \%$ accurate also, and helical CT then suddenly dropped to low accuracy rates of approximately $75 \%$ in four comparative studies. ${ }^{17-20}$ Similar observations have been made in many other areas-for example, recently after the establishment of positron emission tomography for the differential diagnosis of pancreatic tumours. ${ }^{21-23}$ A considerable bias is therefore introduced with each new method when the examiners concerned wish to demonstrate that it is superior to previously established procedures.

These phenomena can probably be explained, at least in part, by methodological differences between the various studies. Small patient numbers, patient selection, the parameters chosen for evaluation, prospective or retrospective analysis, and blindedness in particular, are important factors, to name a few. The examiner's expectations certainly play a crucial role, and these are supported by the clinical impression and the results of other imaging tests conducted prior to the test under consideration. Our results shed light on various aspects of this question. Firstly, the accuracy of EUS (approximately 75\%) in routine clinical conditions was less spectacular than previously reported, particularly in the case of oesophagogastric tumours. Secondly, when the investigator was blinded, there was a substantial reduction in the accuracy rate to approximately 50-60\%, with little difference between the three types of tumours. However, adding the information from one other imaging test (endoscopy or CT) increased the accuracy again by $15-20 \%$, to almost the same level as in routine clinical conditions. These observed effects were not evident in the case of gastric cancer, where the accuracy rates did not improve. However, the reason for this may be a systematic error in the T staging of gastric cancers, as previously discussed, ${ }^{9}$ related to the difficult differentiation between subserosal (T2) and serosal (T3) infiltration and a tendency to overstage ulcerated and proximal gastric tumours. ${ }^{24}$

Other factors that may explain the discrepancies between the fairly moderate clinical results in this study and those of previous publications are related to several types of biases. At first, publication bias, the selective reporting of studies featuring positive or extreme results, may add to this phenomenon. This has recently been debated. ${ }^{25-27}$ Secondly, there may be some case selection bias. It is well known from literature surveys that the best EUS results are usually achieved in cases of advanced cancer. ${ }^{72} 2428$ In study populations in which the majority of patients have T3 cancers, the overall results are therefore likely to be better than in a study such as the present one in which T3 cancers made up less than half of the cases. For example, in a survey published in 1995, 73\% of the tumours included in studies of oesophageal cancer staging using EUS were T3/T4 lesions. ${ }^{29}$ This effect of improving the overall results due to analysing considerably more patients with advanced cancers is also evident in our own data: the sensitivity of EUS for staging Tl/T2 tumours was considerably lower in comparison with the sensitivity for correctly diagnosing more advanced tumours. A further bias may be institutional where initial reports may come from centres of excellence which do not reflect the value of the method in clinical practice-taking into account the learning curve for EUS - ${ }^{30-32}$ but there are few objective data on this in the literature.

To the best of our knowledge, this is the first study in which the accuracy of an imaging procedure such as EUS in the T staging of upper gastrointestinal tract tumours has been assessed not only in a clinical setting but also by blinding the investigator to the clinical findings and other tests, thereby ensuring a more scientific approach in order to test the "real" efficacy of EUS. Reviewing videotape sequences may be inferior to assessment at the live examination; however, well documented tape sequences were selected in which the tumour was fully visualised and examined according to a standardised approach. In addition, a potentially confounding effect of interobserver variability in explaining the divergent results obtained by different methods of data assessment is quite unlikely, in our opinion, as (at least in oesophagogastric tumours where this was tested) the same phenomenon was observed with a second investigator.

The data presented here suggest that the best methodology for evaluating imaging tests is a matter that requires debate. Strictly blinded conditions may yield the scientifically most valid data but at the same time create an artificial situation as in clinical practice the examiner always sees the patient and ideally also takes his or her history. Complementary tests may also be reviewed prior to EUS, and mistakes or complications often have to be avoided. On the other hand, in clinical conditions, these additional data obviously have a significant influence on the performance of the test under consideration (EUS in the present case) so that "true" accuracy rates cannot really be expected. We have therefore also suggested previously that a distinction needs to be made between "scientific" and "clinical" accuracy. ${ }^{5}$ The consensus regarding the "scientific" value of a method also has an impact on clinical case management as it is used to prevent underdiagnosis, overdiagnosis, or misdiagnosis resulting from overestimation of the effectiveness of a single method. The methodology used in studies should be made fully explicit in all cases so that the circumstances in which the respective results were achieved can be recognised.

\section{ACKNOWLEDGEMENT}

We thank M Robertson for his assistance with the English language of the manuscript.

\section{Authors' affiliations}

\section{A Meining, H J Dittler,} Siewert, M Classen, $\mathrm{H}$ Höfler, T Rösch, Departments of Internal Medicine II, Surgery, and Pathology, Klinikum rechts der Isar, Technical University of Munich, Germany

\section{REFERENCES}

1 Rösch T, editor. Endoscopic ultrasonography: state of the art 1995, part 2. Gastrointest Endosc Clin N Am 1995:5:699-849.

2 Canon ME, Carpenter SL, Elta GH, et al. EUS compared with CT, magnetic resonance imaging, and angiography and the influence of biliary stenting on staging accuracy of ampullary neoplasms. Gastrointest Endosc 1999;50:27-33.

3 Palazzo L. Staging of pancreatic carcinoma by endoscopic ultrasonography. Endoscopy 1998;30(suppl 1):A103-7.

4 Gress FG, Hawes RH, Savides TJ, et al. Role of EUS in the preoperative staging of pancreatic cancer: a large single-center experience. Gastrointest Endosc 1999;50:786-91.

5 Rösch T, Dittler HJ, Strobel K, et al. Endoscopic ultrasound criteria for vascular invasion in the staging of cancer of the head of the pancreas: a blind reevaluation of videotapes. Gastrointest Endosc 2000;52:469-77.

6 Ahmad NA, Lewis JD, Ginsberg GG, et al. Endoscopic ultrasound in preoperative staging of pancreatic cancer. Gastrointest Endosc 2000;52:463-8.

7 Rösch T. Staging of pancreatic cancer: analysis of literature results. Gastrointest Endosc Clin N Am 1995;5:735-9.

8 Dufour B, Zins M, Vilgrain V, et al. Comparison between spiral X-ray computed tomography and endosonography in the diagnosis and staging of adenocarcinoma of the pancreas: clinical preliminary study. Gastroenterol Clin Biol 1997;21:124-30. 
9 Rösch T, Classen M. Gastroenterologic endosonography. Stuttgart: Thieme, 1992

10 Howard TJ, Chin AC, Strobe EW, et al. Value of helical computed tomography, angiography, and endoscopic ultrasound in determining resectability of periampullary carcinoma. Am J Surg 1997;174:237-41.

11 Legmann $P$, Vignaux $O$, Dousset $B$, et al. Pancreatic tumors: comparison of dual-phase helical CT and endoscopic sonography. AJR Am J Roentgenol 1998;170:1315-22.

12 Rösch T. Endoscopic ultrasound. Endoscopy 1992;24:144-53.

13 Brugge WR, Lee M, Carey RW, et al. Endoscopic ultrasound criteria for esophageal cancer. Gastrointest Endosc 1997;45:147-52.

14 Freeny PC, Marks WM, Ryan JA, et al. Pancreatic ductal adenocarcinoma: diagnosis and staging with dynamic CT. Radiology 1988;166:125-33

15 Gulliver DJ, Baker ME, Cheng CA, et al. Malignant biliary obstruction: efficacy of thin-section dynamic CT in determining resectability. AJR Am J Roentgenol 1992;159:503-7.

16 DiehI SJ, Lehmann KJ, Sadick M, et al. Pancreatic cancer: value of dual-phase helical CT in assessing resectability. Radiology 1998;206:373-8.

17 Irie $\mathbf{H}$, Honda $\mathrm{H}$, Kaneko $\mathrm{K}$, et al. Comparison of helical CT and MR imaging in detecting and staging small pancreatic adenocarcinoma. Abdom Imaging 1997;22:429-33.

18 Trede $M$, Rumstadt B, Wendl K, et al. Ultrafast magnetic resonance imaging improves the staging of pancreatic tumors. Ann Surg 1997; 226:393-407.

19 Gorelick AB, Scheiman JM, Fendrick AM. Identification of patients with resectable pancreatic cancer: at what stage are we? Am J Gastroenterol 1998;93:1995-6.

20 Sheridan MB, Ward J, Guthrie JA, et al. Dynamic contrastenhanced MR imaging and dual-phase helical CT in the preoperative assessment of suspected pancreatic cancer: a comparative study with receiver operating characteristic analysis. AJR Am J Roentgenol 1999; 173:585-90.
21 Inokuma T, Tamaki N, Torizuka T, et al. Evaluation of pancreatic tumors with positron emission tomography and F-1 8 fluorodeoxyglucose: comparison with CT and US. Radiology 1995;195:345-52.

22 Friess $\mathbf{H}$, Langhans J, Ebert $M$, et al. Diagnosis of pancreatic cancer by 2[18F]-fluoro-2-deoxy-D-glucose positron emission tomography. Gut $1995 ; 36: 771-7$

23 Sendler A, Avril N, Helmberger $\mathrm{H}$, et al. Preoperative evaluation of pancreatic masses with positron emission tomography using 18F-fluorodeoxyglucose: diagnostic limitations. World J Surg 2000;24:1 121-9

24 Salminen JT, Färkkilä, Rämö OJ, et al. Endoscopic ultrasonography in the preoperative staging of adenocarcinoma of the distal oesophagus and oesophagogastric junction. Scand J Gastroenterol 1999:34:1178-82

25 Eloubeidi MA, Wade SB, Provenzale D. Factors associated with acceptance and full publication of $\mathrm{Gl}$ endoscopic research originally published in abstract form. Gastrointest Endosc 2001;53:275-82.

26 Shaheen NJ, Crosby MA, Bozymski EM, et al. Is there publication bias in the reporting of cancer risk in Barrett's esophagus? Gastroenterol 2000;119:333-8

27 Cloft HJ, Shengelaia GG, Marx WF, et al. Preliminary reports and the rates of publication of follow-up reports in peer-reviewed, indexed journals. Acad Med 2001;76:638-4.

28 Heidemann J, Schilling MK, Schmassmann A, et al. Accuracy of endoscopic ultrasonography in preoperative staging of esophageal carcinoma. Dig Surg 2000;17:219-24.

29 Rösch T. Endosonographic staging of esophageal cancer: a review of literature results. Gastrointest Endosc Clin N Am 1995;5:537-47.

30 Carmody BJ. Otchy: learning curve of transrectal ultrasound. Dis Colon Rectum 2000:43:193-7.

31 Fockens $\mathbf{P}$, Van den Brande JH, van Dullemen $\mathrm{HM}$, et al Endosonographic T-staging of esophageal carcinoma: a learning curve. Gastrointest Endosc 1996;44:58-62.

32 Schlick T, Heintz A, Junginger T. The examiner's learning effect and its influence on the quality of endoscopic ultrasonography in carcinoma of the esophagus and gastric cardia. Surg Endosc 1999;13:894-8.

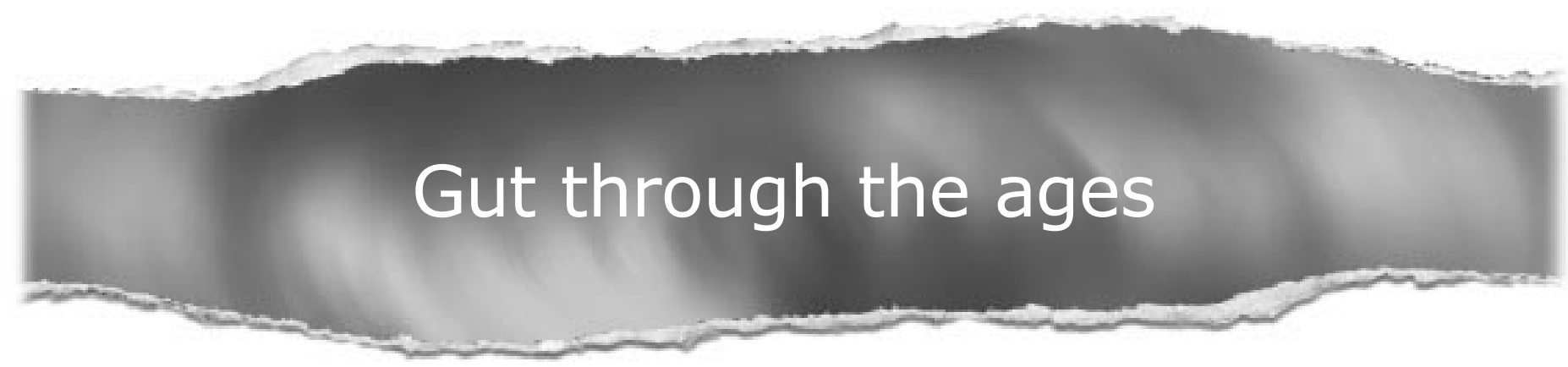

\section{Browse the Archive}

Gut online has an archive of content dating back to 1966.

Full text from 1997; abstracts from 1975; table of contents from 1966

\section{www.gutjnl.com}

